\title{
Ultrasound enhanced Azo Dye adsorption by Graphene Oxide Nanocomposite
}

\author{
Priya Banerjee ${ }^{\star}$, Aniruddha Mukhopadhyay ${ }^{1}$ and Papita Das ${ }^{2}$ \\ IDepartment of Environmental Science, University of Calcutta, 35, Ballygunge Circular Road, Kolkata - 700 019, India \\ 2 Department of Chemical Engineering, Jadavpur University, 188, Raja S.C. Mullick Road, Kolkata - 700 032, India
}

\section{Article Info

*Corresponding author:
Priya Banerjee
Ph.D. Fellow
Department of Environmental Science
University of Calcutta
35 BallygungeCicular Road
Kolkata - 700019
India
Tel: +919874803469
E-mail: prya_bnrje@yahoo.com

Received: September 1, 2018

Accepted: September 7, 2018

Published: September 13, 2018

Citation: Banerjee P, Mukhopadhayay A, Das P. Ultrasound enhanced Azo Dye adsorption by Graphene Oxide Nanocomposite. Madridge J Nanotechnol Nanosci. 2018; 3(2): 106-111. doi: $10.18689 / m j n n-1000120$

\begin{abstract}
Copyright: @ $₫ 2018$ The Author(s). This work is licensed under a Creative Commons Attribution 4.0 International License, which permits unrestricted use, distribution, and reproduction in any medium, provided the original work is properly cited.
\end{abstract}

Published by Madridge Publishers

\begin{abstract}
Reactive azo dyes appearing in almost all streams of wastewater reportedly possess carcinogenic and mutagenic properties and are often biorecalcitrant in nature. In recent years, the process of adsorption has received much significance as one of the most efficient, easy to operate and cost effective procedures implemented for treatment of effluents containing different types of dyes. The present study was performed to investigate the potential of Graphene oxide (GO) - Zinc oxide nanoparticle (ZnO NPs) composites as an inexpensive, convenient and non-toxic adsorbent for removal of Methylene blue (MB) from solution. The ZnO NPs used in this study were synthesized in a green route from leaf extracts of basil (Ocimum sanctum). The adsorption phenomenon was catalysed by ultrasound to achieve higher rates of removal in significantly reduced time. The process was optimized using Response Surface Methodology. Under optimized conditions, 99.87\% removal of upto $120 \mathrm{ppm}$ dye was achieved in 6 min using only $0.75 \mathrm{~g}$ $\mathrm{L}^{-1} \mathrm{GO}-\mathrm{ZnO}$ NP nanocomposite as adsorbent. Characterization of this nanocomposite (using Electron Microscopy and Fourier Transform Infrared Spectroscopy) depicted changes in the surface morphology and chemical properties of the adsorbent resulting from MB uptake. Antibacterial properties of the GO-ZnONP nanocomposite was also determined and found to be higher than its individual components. Results indicated that the fabricated adsorbent was highly efficient and may be investigated further for treatment of industrial or domestic effluents.
\end{abstract}

Keywords: Azo dye; Graphene oxide; Nanocomposites; Ultrasound aided dye adsorption; Response Surface Methodology; Adsorbent characterization.

\section{Introduction}

Alarming proportions of azo dyes present in industrial effluents exert a severe impact on the environment by causing harm to humans and ecosystems adjacent to their point of discharge $[1,2] .7 \times 10^{5}$ tonnes of dyes are approximately manufactured commercially every year for industrial applications [3]. These dyes are reckoned as primary toxicants due to their potentials of causing carcinogenesis and mutagenesis [4]. Therefore it has become highly essential to ensure efficient removal azo dyes from effluents bearing them in order to maintain health and safety of the environment [4].

Of all other methods of effluent treatment reported till date for treatment of dye compounds present in effluents, adsorption has been widely reckoned as the most efficient due to its applicability for continuous effluent treatment, low cost and nonrequirement of harsh chemicals and organic solvents [2-5]. Recent investigations have reported graphene oxide (GO) as a highly suitable adsorbent due to its benign nature, simple synthesis, defined and homogenous structure and good stability [2]. However, $\mathrm{GO}$ sheets tend to aggregate on being suspended in aqueous media rendering the 
same unsuitable for adsorption by reducing its potential for reuse [4-6]. In order to overcome this limitation of GO, recent studies have reported insertion of nanoparticles between adjacent $\mathrm{GO}$ layers for reducing aggregation and obtaining greater surface area for adsorption [6]. Incorporation of nanoparticles were found to widen the space between adjacent $\mathrm{GO}$ sheets and facilitate better fluid percolation [6].

In the present study composites were prepared using GO and $\mathrm{ZnO}$ nanoparticles ( $\mathrm{ZnO} \mathrm{NPs}$ ) and investigated for adsorption of cationic azo dye methylene blue (MB). Ultrasound irradiation was applied throughout the treatment for accelerating the process of adsorption and in turn facilitating higher uptake of dye by the $\mathrm{GO}$ composite with significantly reduced duration of treatment and cost [4]. The composites were characterized using scanning electron microscopy (SEM), transmission electron microscopy (TEM) and Fourier transform infrared spectroscopy (FTIR). Determination of the effect of interaction of significant process parameters (like initial dye concentration, solution $\mathrm{pH}$, ambient temperature, adsorbent dosage and duration of exposure to ultrasound irradiation) on $\mathrm{MB}$ uptake by $\mathrm{GO}-\mathrm{ZnO}$ nanocomposite and process optimization were elucidated with Central Composite Design (CCD) scheme of Response Surface Methodology (RSM), an integrated mathematical and statistical tool.

\section{Experimental Methodology}

\section{Materials and equipment}

All chemicals (analytical grade) used here in were commercially obtained from Merck, India and used as received. Stock solutions $\left(100 \mathrm{mg} \mathrm{L}^{-1}\right)$ of MB $\left(\boldsymbol{\lambda}_{\max }=664 \mathrm{~nm}\right)$ were prepared using distilled water and diluted with the same for obtaining working solutions of desired concentrations. All through the study, solution $\mathrm{pH}$ was adjustedusing $0.1 \mathrm{~N} \mathrm{HCl}$ or $0.1 \mathrm{M} \mathrm{NaOH}$ as required.

Ultrasonic irradiation was provided using an ultransonic bath (Rivotek, India) having a heating system which was operated at a frequency of $30 \pm 3 \mathrm{kHz}$ with a power of $120 \mathrm{~W}$ for performing ultrasound aided $\mathrm{MB}$ adsorption. Residual $\mathrm{MB}$ concentrations at any point was determined with an UV-VIS spectrophotometer (Perkin Elmer $\lambda$ 35, USA) using a quartz cuvette. The GO-ZnO nanocomposites were characterized by SEM (ZEISSEVO-MA10, Germany) after gold-coating the samples with a sputter coater. Nanocomposites were also analysed using a TEM (JEOL, Japan; Model No.JEM2100h) using samples cast and air dried on carbon coated copper grids. Chemical analysis of the GO-ZnO nanocomposites was conducted with a FTIR (JascoFTIR-6300, Japan) in transmittance mode via the $\mathrm{KBr}$ pellet method. Samples pelleted using $\mathrm{KBr}$ (FTIR grade) were scanned over a range of $400-4000 \mathrm{~cm}^{-1}$.

\section{Preparation of GO-ZnO NP nanocomposite}

GO used in this study was prepared by modified Hummers method as reported by Banerjee et al [6]. ZnO NPs used in this study were synthesised in a green route reported by Devi and Gayathri using the leaf extracts of Basil (Ocimum sanctum) [7].
For synthesis of the GO-ZnO NP composite, $5 \mathrm{~g}$ GO was suspended in $20 \mathrm{~mL}$ ethanol and exposed to ultrasonic irradiation for $2 \mathrm{~h}$. The $\mathrm{ZnO}$ nanoparticles $(5 \mathrm{~g})$ prepared previously were suspended into a mixture of methanol $(18 \mathrm{~mL})$ and chloroform $(2 \mathrm{~mL})$ and separately exposed to ultrasonic irradiation for $2 \mathrm{~h}$.at the end of $2 \mathrm{~h}$, the $\mathrm{GO}$ and $\mathrm{ZnO}$ suspensions were mixed and subjected to constant stirring for $24 \mathrm{~h}$.The composite was then separated by centrifugation, washed using methanol (several times), dried, ground and stored for further studies.

\section{RSM (CCD) study}

For optimization of ultrasound aided $\mathrm{MB}$ adsorption by $\mathrm{GO}-\mathrm{ZnO}$, a five factor based model was developed using RSM (CCD) with Design Expert, Version 7.0 (Minneapolis, USA). 50 experiments suggested by this model were carried out in this study. The ranges, levels and design matrix of selected parameters have been given in Table 1. Performance target and ramp were kept constant for ensuring best results.

Table 1. Computational details of Response Surface Methodology (CCD) for MB removal using GO-ZnO NP composite in presence of ultrasound irradiation

\begin{tabular}{|l|c|c|c|c|c|c|}
\hline \multirow{2}{*}{ Factors } & \multirow{2}{*}{ Units } & \multicolumn{3}{|c|}{ Levels } & \multicolumn{3}{c|}{ Star point $\alpha=2.38$} \\
\cline { 3 - 7 } & & Low (-1) & Central (0) & High (+1) & $-\alpha$ & $+\alpha$ \\
\hline Initial MB Conc. & $\mathrm{mg} \mathrm{L}^{-1}$ & 57.07 & 90.70 & 124.34 & 10.7049 & 170.705 \\
\hline $\mathrm{pH}$ & - & 4.8 & 8.0 & 11.2 & 0.51366 & 15.51366 \\
\hline Temperature & ${ }^{\circ} \mathrm{C}$ & 25.0 & 32.5 & 40.0 & 14.66189 & 50.33811 \\
\hline Adsorbent Dose & $\mathrm{g} \mathrm{L}^{-1}$ & 0.63 & 0.89 & 1.15 & 0.266905 & 1.516905 \\
\hline $\begin{array}{l}\text { Ultrasound Exposure } \\
\text { Duration }\end{array}$ & $\min$ & 4.8 & 8.0 & 11.2 & 0.51366 & 15.51366 \\
\hline
\end{tabular}

The empirical association between the selected independent variables was determined by the quadratic polynomial equation given as follows: $Y=m_{0}+\sum_{a=1}^{c=5} m_{a} x_{a}+\sum_{a=1}^{c=5} \sum_{b=1}^{c=5} m_{a b} x_{a} x_{b}+\sum_{a=1}^{c=5} m_{a a} x_{a}^{2}+\varepsilon \ldots(5)$

Whereby, $Y, m_{0}, m_{p}\left(p=a, b, a b\right.$, etc.), $x_{q}(q=a, b$, etc.) and represent dependent variable (response), constant coefficients, regression coefficients (linear, quadratic and interaction respectively), independent variables (experimental parameters) and error respectively. 50 experiments suggested by RSM (CCD) were replicated thrice for reducing handling error. Optimized experimental parameters were derived using Derringer's desirability function [4]. The applied goals were maintained within range and at a maximum for experimental parameters and responses respectively.

\section{Reusability study}

Reusability potential of adsorbents are of high significance for investigat.ions related to adsorption, desorption and adsorbate recovery. On attaining its maximum adsorption capacity, the adsorbents were retrieved by centrifugation and subjected to treatment with $4.0 \mathrm{M} \mathrm{NaOH}$ for regeneration after complete desorption. The regenerated adsorbents were re-applied for MB adsorption under optimized conditions. This processes of adsorption and desorption were repeated for ten consecutive cycles.

\section{Statistical analysis}

All experiments were repeated thrice and the results have been presented as Mean \pm SD. Statistical significance was calculated with ANOVA using Design Expert, Version 7.0, USA. 


\section{Results and Discussion}

\section{RSM (CCD) analysis}

The output (\% MB removal) of the 50 combinations of experimental parameters suggested by the RSM (CCD) has been shown in Table 2. A large F value (442.57) indicated the high significance $(p<0.0001)$ of the quadratic model predicted by RSM and also established that the quadratic polynomial equation guiding this process was significant at $95 \%$ confidence level. The insignificant lack of fit $(>0.05)$ also indicated the validity of the quadratic model for the present study. The considerable agreement between the regression coefficient $R^{2}(0.995)$, the adjusted $R^{2}(0.992)$ and the predicted $R^{2}$ (0.981) for $M B$ uptake suggested appropriateness of the quadratic polynomial model for predicting the output of the concerned process.

Table 2. Experiments suggested by Response Surface Methodology (CCD) for MB removal using GO-ZnO NP composite in presence of ultrasound irradiation and their respective responses

\begin{tabular}{|c|c|c|c|c|c|c|}
\hline Run & $\begin{array}{c}\text { Initial MB } \\
\text { conc. } \\
\text { (mg L-1) }\end{array}$ & pH & $\begin{array}{c}\text { Temperature } \\
\text { ('C) }\end{array}$ & $\begin{array}{c}\text { Adsorbent } \\
\text { dose } \\
\text { (g L-1) }\end{array}$ & $\begin{array}{c}\text { USI } \\
\text { duration } \\
\text { (min) }\end{array}$ & $\begin{array}{c}\text { MB } \\
\text { removal } \\
\text { (\%) }\end{array}$ \\
\hline 1 & 90.70 & 8.0 & 32.5 & 0.89 & 15.5 & 62.87 \\
\hline 2 & 57.07 & 11.2 & 25 & 1.15 & 11.2 & 82.84 \\
\hline 3 & 57.07 & 11.2 & 40 & 0.63 & 4.9 & 72.32 \\
\hline 4 & 57.07 & 4.9 & 25 & 0.63 & 11.2 & 34.12 \\
\hline 5 & 124.34 & 11.2 & 25 & 0.63 & 11.2 & 66.12 \\
\hline 6 & 124.34 & 4.9 & 40 & 0.63 & 11.2 & 54.36 \\
\hline 7 & 90.70 & 8.0 & 32.5 & 0.27 & 8.0 & 62.72 \\
\hline 8 & 124.34 & 11.2 & 40 & 1.15 & 4.9 & 90.15 \\
\hline 9 & 57.07 & 11.2 & 25 & 0.63 & 11.2 & 62.14 \\
\hline 10 & 90.70 & 8.0 & 32.5 & 0.89 & 8.0 & 97.72 \\
\hline 11 & 10.70 & 8.0 & 32.5 & 0.89 & 8.0 & 75.14 \\
\hline 12 & 90.70 & 14.0 & 32.5 & 0.89 & 8.0 & 75.14 \\
\hline 13 & 90.70 & 8.0 & 32.5 & 0.89 & 8.0 & 99.72 \\
\hline 14 & 124.34 & 11.2 & 40 & 1.15 & 11.2 & 99.81 \\
\hline 15 & 57.07 & 4.9 & 25 & 0.63 & 4.9 & 21.32 \\
\hline 16 & 57.07 & 11.2 & 40 & 1.15 & 4.9 & 87.14 \\
\hline 17 & 124.34 & 11.2 & 40 & 0.63 & 11.2 & 99.12 \\
\hline 18 & 57.07 & 11.2 & 25 & 0.63 & 4.9 & 38.11 \\
\hline 19 & 124.34 & 4.9 & 40 & 0.63 & 4.9 & 42.44 \\
\hline 20 & 124.34 & 11.2 & 25 & 1.15 & 11.2 & 77.14 \\
\hline 21 & 124.34 & 4.9 & 25 & 0.63 & 4.9 & 12.98 \\
\hline 22 & 57.07 & 4.9 & 40 & 0.63 & 11.2 & 55.24 \\
\hline 23 & 57.07 & 4.9 & 25 & 1.15 & 11.2 & 30.31 \\
\hline 24 & 57.07 & 4.9 & 40 & 1.15 & 11.2 & 38.33 \\
\hline 25 & 90.70 & 8.0 & 50.3 & 0.89 & 8.0 & 87.15 \\
\hline 26 & 170.70 & 8.0 & 32.5 & 0.89 & 8.0 & 64.24 \\
\hline 27 & 90.70 & 8.0 & 32.5 & 0.89 & 8.0 & 99.77 \\
\hline 28 & 124.34 & 4.9 & 25 & 0.63 & 11.2 & 26.17 \\
\hline 29 & 57.07 & 4.9 & 25 & 1.15 & 4.9 & 31.66 \\
\hline 30 & 90.70 & 2.0 & 32.5 & 0.89 & 8.0 & 0.54 \\
\hline 31 & 124.34 & 4.9 & 40 & 1.15 & 4.9 & 27.55 \\
\hline 32 & 90.70 & 8.0 & 32.5 & 0.89 & 8.0 & 99.72 \\
\hline 33 & 90.70 & 8.0 & 32.5 & 0.89 & 8.0 & 99.72 \\
\hline 34 & 57.07 & 4.9 & 40 & 1.15 & 4.9 & 37.12 \\
\hline 35 & 90.70 & 8.0 & 32.5 & 0.89 & 8.0 & 99.72 \\
\hline & & & & & & \\
\hline 32 & & & & & & \\
\hline
\end{tabular}

\begin{tabular}{|l|c|c|c|c|c|c|}
\hline 36 & 90.70 & 8.0 & 32.5 & 0.89 & 8.0 & 99.72 \\
\hline 37 & 124.34 & 4.9 & 40 & 1.15 & 11.2 & 28.48 \\
\hline 38 & 90.70 & 8.0 & 32.5 & 0.89 & 0.5 & 24.66 \\
\hline 39 & 124.34 & 11.2 & 25 & 0.63 & 4.9 & 40.19 \\
\hline 40 & 124.34 & 4.9 & 25 & 1.15 & 11.2 & 22.16 \\
\hline 41 & 57.07 & 11.2 & 25 & 1.15 & 4.9 & 62.18 \\
\hline 42 & 90.70 & 8.0 & 14.6 & 0.89 & 8.0 & 27.11 \\
\hline 43 & 57.07 & 11.2 & 40 & 0.63 & 11.2 & 90.12 \\
\hline 44 & 124.34 & 11.2 & 25 & 1.15 & 4.9 & 63.17 \\
\hline 45 & 57.07 & 4.9 & 40 & 0.63 & 4.9 & 43.18 \\
\hline 46 & 124.34 & 11.2 & 40 & 0.63 & 4.9 & 72.11 \\
\hline 47 & 124.34 & 4.9 & 25 & 1.15 & 4.9 & 17.18 \\
\hline 48 & 90.70 & 8.0 & 32.5 & 0.89 & 8.0 & 99.72 \\
\hline 49 & 90.70 & 8.0 & 32.5 & 1.52 & 8.0 & 77.14 \\
\hline 50 & 57.07 & 11.2 & 40 & 1.15 & 11.2 & 95.22 \\
\hline
\end{tabular}

The $A_{\text {deq }}$ Precision (60.07) indicated that the selected model determined \% MB removal in the semi-empirical equation given below.

\% MB Removal (Predicted) $=-409.00672+0.58278$ * Initial $\mathrm{MB}$ conc. +20.57580 * $\mathrm{pH}+10.85249$ * Temperature +187.90975 * Adsorbent dose +19.56903 * USI duration +0.022893 *Initial MB conc. ${ }^{*} \mathrm{pH}+4.07799 \mathrm{E}-003$ * Initial MB conc. * Temperature -0.12763 * Initial MB conc. * Adsorbent dose $+3.62392 \mathrm{E}-003$ * Initial MB conc. * USI duration $+0.11007 * \mathrm{pH}$ * Temperature +6.57854 * $\mathrm{pH}$ * Adsorbent dose +0.28724 * $\mathrm{pH}$ * USI duration -1.75337 * Temperature * Adsorbent dose -0.033747 * Temperature * USI duration3.26589 * Adsorbent dose * USI duration -4.74479E-003 * Initial MB conc. ${ }^{2}-1.73474$ * $\mathrm{pH}^{2}-0.13491$ * Temperature $^{2}$ -77.12424 * Adsorbent dose 2 -1.00074 * USI duration ${ }^{2}$

The response values predicted using this equation was in good accord with that obtained experimentally (as shown in Figure 1).
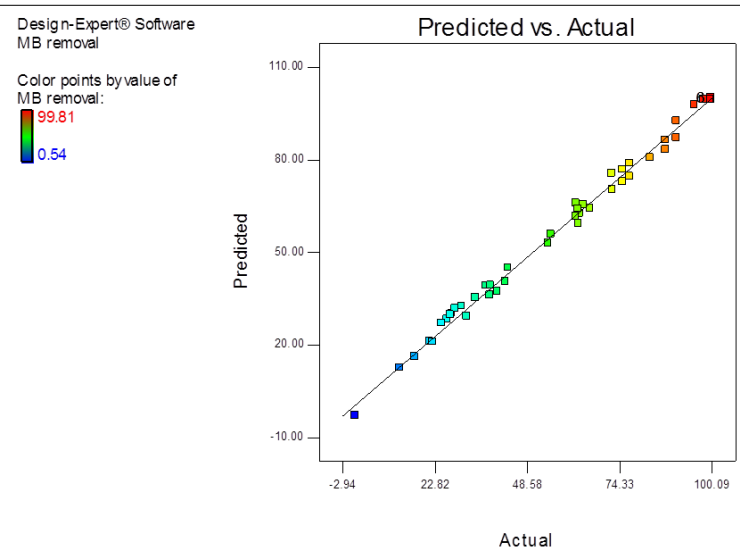

Figure 1. Graph showing comparison between \% dye removal predicted by RSM (CCD) analysis and \% dye removal obtained experimentally for MB adsorption using GO- ZnO NP composite

3D surface plots representing inter-parameter interactions effecting ultrasound aided MB adsorption by GO-ZnO NP composite have been shown in Figures 2 and 3. The significant inter-parameter interactions $(p<0.0001)$ indicated by ANOVA analysis has been discussed as follows. 


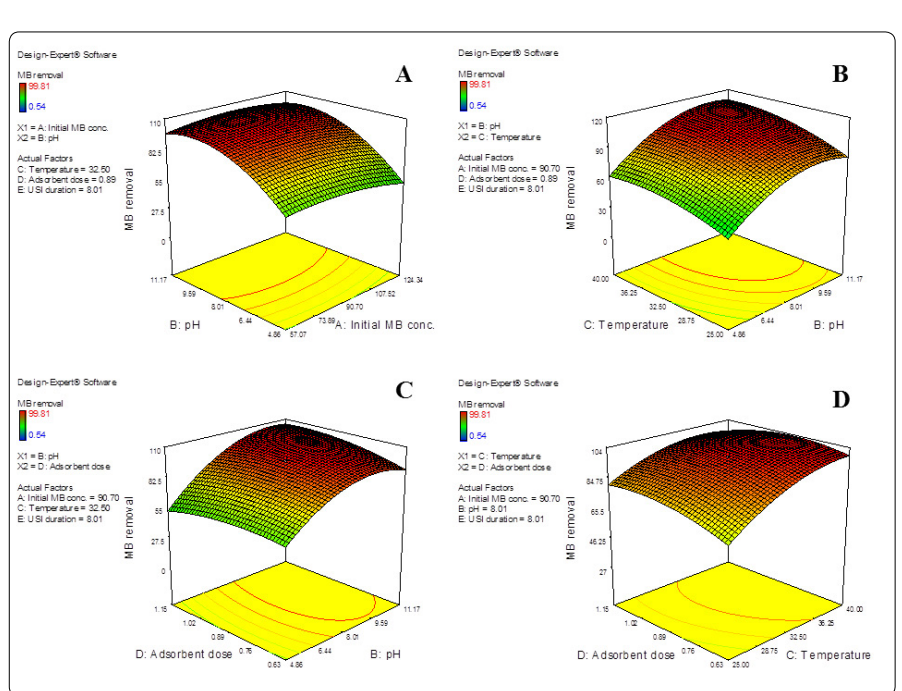

Figure 2. 3D plots obtained from RSM (CCD) analysis showing effect of independent variables on MB adsorption using GO- ZnO NP composite. Figures $A-D$ represent interactions of varying initial $M B$ concentration and solution $\mathrm{pH}$, solution $\mathrm{pH}$ and ambient temperature, solution $\mathrm{pH}$ and adsorption dosage and adsorption dosage and ambient temperature respectively on the process of adsorption reported in this study.

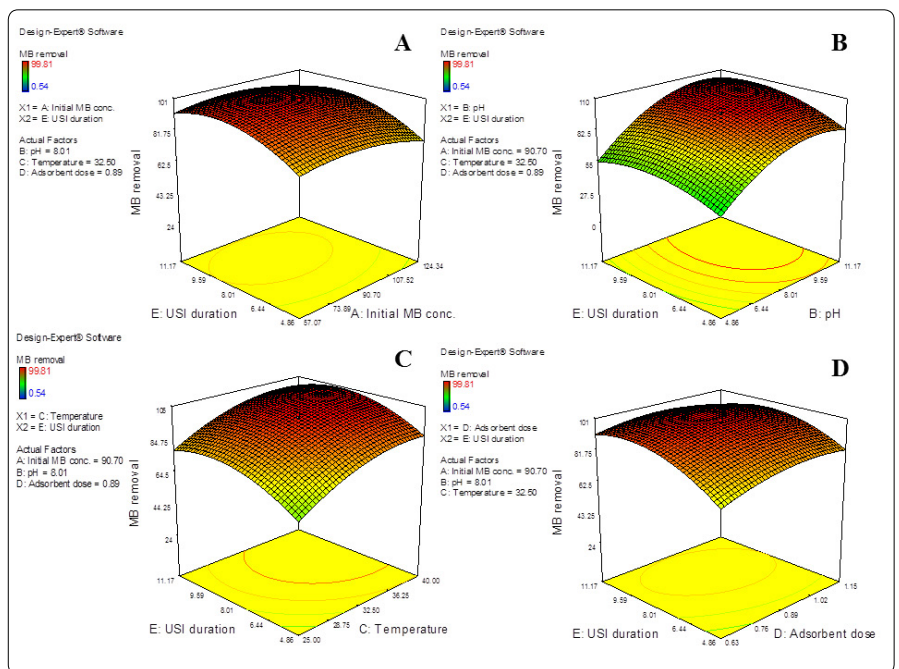

Figure 3. 3D plots obtained from RSM (CCD) analysis showing effect of independent variables with respect to duration of ultrasound irradiation

(USI) on MB adsorption using GO- ZnO NP composite. Figures A-D

represent interactions of duration of USI with varying initial MB

concentration, solution $\mathrm{pH}$, ambient temperature and adsorption dosage respectively on the process of adsorption reported in this study.

\section{Initial MB conc. vs. $\mathbf{p H}$}

As evident from Figure $2 \mathrm{~A}, \mathrm{MB}$ uptake by GO-ZnO NP composite was found to rise with a parallel increase in solution $\mathrm{pH}$ and initial dye concentration till attaining $\mathrm{pH}$ 8. However, $\%$ dye removal was found to decline with further rise in solution $\mathrm{pH}$. This may have had occurred due to weakening of attractive forces between cationic dye moieties and adsorbent surface under basic $\mathrm{pH}$ conditions [6-8].

\section{Ambient temperature vs. solution $\mathbf{p H}$}

As evident from Figure 2B, MB uptake was found to increase with a corresponding rise in both ambient temperature and solution $\mathrm{pH}$ (till $\mathrm{pH}$ 8). Increase in temperature may have enhanced porosity and pore volume in turn facilitating higher dye adsorption [4].

\section{Adsorbent dosage vs. solution $\mathbf{p H}$}

As evident from Figure $2 C, M B$ uptake was found to increase with a corresponding rise in both adsorbent dosage and solution $\mathrm{pH}$ (till $\mathrm{pH}$ 8). Higher adsorbent dosage may have provided greater surface area with higher number of adsorption sites thereby enabling better rates of dye uptake $[2,4,9]$.

\section{Adsorbent dosage vs. ambient temperature}

As shown in Figure 2D, dye uptake was found to increase with a corresponding rise in both adsorbent dosage and ambient temperature. Diffusion of dye molecules at higher temperatures and increased adsorptive surface area may have had facilitated higher uptake of MB by GO-ZnO NP composites [10].

\section{Duration of ultrasonic irradiation application vs. other significant process parameters}

Effect of duration of ultrasonic irradiation in combination with parameters like initial dye concentration, solution $\mathrm{pH}$ temperature and adsorbent dosage has been shown as Figure 3 (A-D) respectively. It was observed that dye uptake had improved with a corresponding increase in all tested parameters. The significantly increased rates of adsorption achieved over highly reduced duration of treatment could be attributed to robust effects of ultrasound irradiation exerted on mass transfer [11]. Exposure to shock waves and drastic micro jets arising from violent collapse of bubbles formed at the adsorbent-adsorbate interface due to cavitation may have had caused enhanced MB uptake by GO-ZnO NP composites [4-8]. Increase in MB uptake with parallel increase in temperature also suggested an endothermic nature of the concerned process of adsorption [4].

\section{Optimization using RSM(CCD)}

As determined by the Derringer's desirability function, the optimized conditions favoringmost efficient $M B$ adsorption using the GO- ZnO NP composite were an initial $\mathrm{MB}$ concentration of $124.34 \mathrm{mg} \mathrm{L}^{-1}$, a solution $\mathrm{pH}$ of 8.8 , an ambient temperature of $35.7^{\circ} \mathrm{C}$, an adsorbent dosage of 0.72 $\mathrm{g} \mathrm{L}^{-1}$ and an ultrasound exposure of $8.6 \mathrm{~min}$. A maximum of $99.94 \%$ of MB removal was recorded with these optimized experimental parameters.

\section{Adsorbent characterization}

\section{Electron microscopic analysis}

Images of GO-ZnO Nanoparticle composites (before and after adsorption) recorded using SEM have been shown in Figure 4 ( $A$ and $B$ ) respectively. Figure $4 A$ shows oval ZnO NPs lodged between adjacent $G O$ sheets. Figure $4 B$ shows smoothening of composite surfaces probably arising from adsorption of MB.

Images of GO-ZnO NP composites (before and after adsorption) recorded using TEM have been shown in Figure 4 $(C$ and $D)$ respectively. Images obtained with TEM are similar to those recorded with SEM. 


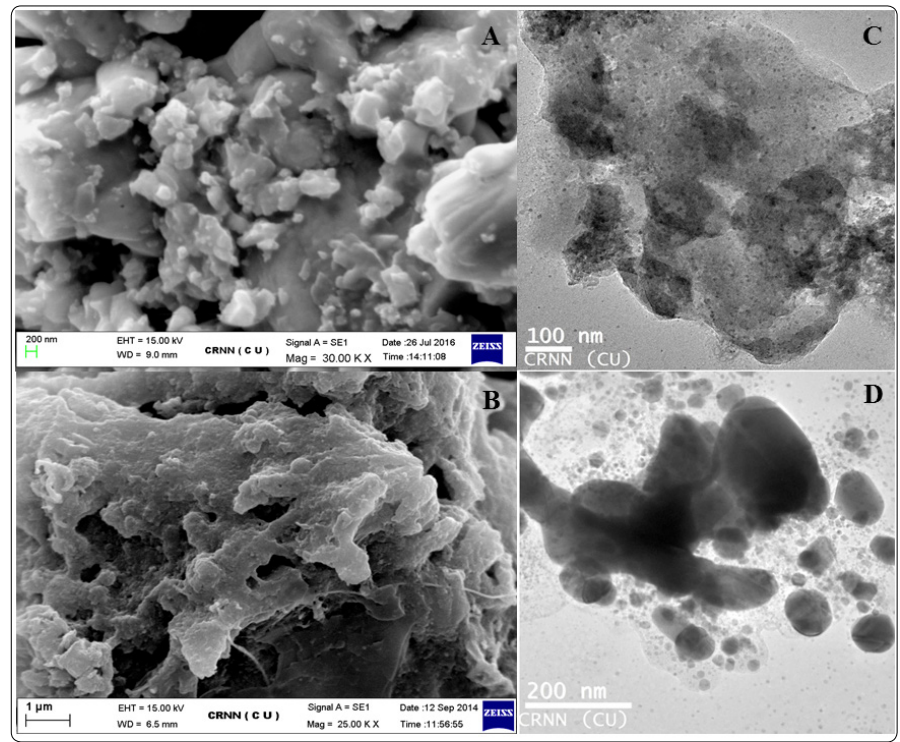

Figure 4. Characterization of GO- ZnO NP composite. Figures A and $B$ reveal SEM images of GO- ZnO NP composite captured before and after adsorption respectively. Figures $A$ and $B$ reveal SEM images of GO- ZnO NP composite captured before and after adsorption respectively.

\section{FTIR analysis}

FTIR analysis of GO-ZnO NP composites (before and after adsorption) has been shown in spectrum $A$ and $B$ of Figure 5 respectively. Spectrum $A$ shows characteristic peaks of $G O$ at $3448.00,1703.80,1612.40$ and $1034.60 \mathrm{~cm}^{-1}$ indicating $-\mathrm{OH}_{\text {, - }}$ $\mathrm{C}=\mathrm{O}$ (carboxyl), $-\mathrm{C}=\mathrm{C}$ and $-\mathrm{C}-\mathrm{O}$ (alkoxy) groups respectively [4]. Spectrum A also revealed characteristic peaks of ZnO NPs at $440.00 \mathrm{~cm}^{-1}$ indicating and $\mathrm{Zn}-\mathrm{O}$ respectively [12]. Besides characteristic peaks of GO and $\mathrm{ZnO} N P s$, additional peaks at $3302.40,1470.40,1250.60$ and $593.96 \mathrm{~cm}^{-1}$ recorded in spectrum $\mathrm{B}$ indicated $-\mathrm{CH}_{3}$ (asymmetric), carbonyl groups and mono-substitution of acetylene benzene derivatives respectively and were considered as characteristic peaks of $\mathrm{MB}$ occurring due to efficient adsorption of the same [13].
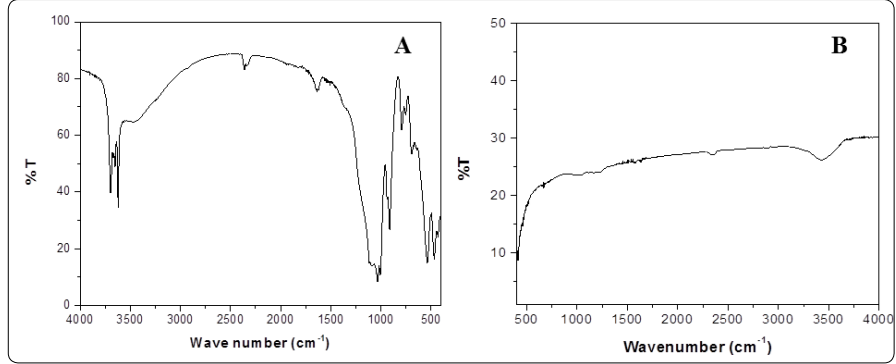

Figure 5. FTIR spectrum of GO- ZnO NP composite before (spectrum A) and after (spectrum B) adsorption.

\section{Reusability potential}

Adsorption efficiency of GO- ZnO NP nanocomposites reported in this study were found to remain unaltered upto eight out of ten adsorption-desorption cycles conducted under optimized conditions. Maximum \% MB removal was found to be $85.62 \%$ and $78.18 \%$ in the last two cycles respectively. This reduction in \% MB removal may have had occurred due to alterations of adsorbent surface arising from repeated alkali exposure or loss of mass encountered during regeneration studies.

\section{Conclusion}

Results obtained in the present study established GO-ZnO NP composite as an effective adsorbent for methylene blue from its aqueous solutions. Ultrasound irradiation was also proved to be a significant tool for accelerating rate of adsorption. The convenient synthesis of GO-ZnO NP composite, its reusability potential, requirement in highly low doses and rapid rate of pollutant uptake rendered the same as an extremely promising adsorbent suitable for wide-scale effluent treatment. Hence, it may be concluded that GO-ZnO NP may be further investigated for treatment of various streams of wastewater.

\section{Conflicts of interest}

The authors hereby declare that they have no conflict of interest.

\section{Acknowledgement}

This study was performed using departmental grants. Authors acknowledge all members of Department of Environmental Science, University of Calcutta for their cooperation and support. Authors also acknowledge Center for Research in Nanoscience and Nanotechnology, University of Calcutta, for proving the SEM, TEM and FTIR facilities used in this study.

\section{References}

1. Roosta M, Ghaedi M, Daneshfar A, Sahraei R, Asghari A. Optimization of the ultrasonic assisted removal of methylene blue by gold nanoparticles loaded on activated carbon using experimental design methodology. Ultrason Sonochem. 2014; 21(1): 242-252. doi: 10.1016/j.ultsonch.2013.05.014

2. Banerjee $P$, Sau $S$, Das $P$, Mukhopadhayay A. Optimization and modelling of synthetic azo dye wastewater treatment using graphene oxide nanoplatelets: characterization toxicity evaluation and optimization using artificial neural network. Ecotoxicol Environ Saf. 2015; 119: 47-57. doi: 10.1016/j.ecoenv.2015.04.022

3. Gupta VK, Suhas. Application of low cost adsorbents for dye removal-a review. J Environ Manage. 2009; 90(8): 2313-2342. doi: 10.1016/j.jenvman.2008.11.017

4. Banerjee $P$, Barman SR, Mukhopadhayay A, Das P. Ultrasound assisted mixed azo dye adsorption by chitosan-graphene oxide nanocomposite. ChemEng Res Des. 2017; 117: 43-56. doi: 10.1016/j.cherd.2016.10.009

5. Liu L, Li C, Bao C, et al. Preparation and characterization of chitosan/ graphene oxide composites for the adsorption of $\mathrm{Au}(\mathrm{III})$ and $\mathrm{Pd}(\mathrm{II})$. Talanta. 2012; 93: 350-357. doi: 10.1016/j.talanta.2012.02.051

6. Banerjee P, Mukhopadhyay A, Das P. Graphene oxide-nanobentonite composite sieves for enhanced desalination and dye removal. Desalination. 2017; doi: 10.1016/j.desal.2017.06.010

7. Devi RS, Gayathri R. Green synthesis of zinc oxide nanoparticles by using Hibiscus rosa-sinensis. Int J Curr Eng Technol. 2014; 4(4): 2444-2446.

8. Jamshidi $\mathrm{M}$, Ghaedi $\mathrm{M}$, Dashtian $\mathrm{K}$, et al. Highly efficient simultaneous ultrasonic assisted adsorption of brilliant green and eosin B onto ZnS nanoparticles loaded activated carbon: artificial neural network modeling and central composite design optimization. SpectrochimActa A Mol Biomol Spectrosc. 2016; 153: 257-267. doi: 10.1016/j.saa.2015.08.024

9. Saeed A, Sharif M, Iqbal M. Application potential of grape fruit peel as dye sorbent: kinetics, equilibrium and mechanism of crystal violet adsorption. $J$ Hazar Mater. 2010; 179(1-3): 564-572. doi: 10.1016/j.jhazmat.2010.03.041

10. Goswami S, Banerjee P, Datta S, Mukhopadhayay A, Das P. Graphene oxide nanoplatelets synthesized with carbonized agro-waste biomass as green precursor and its application for the treatment of dye rich waste water. Proc Safe Environ Prot. 2017; 106: 163-172. doi: 10.1016/j.psep.2017.01.003 
11. Asfaram A, Ghaedi M, Hajati S, Goudarzi A, Bazrafshan AA. Simultaneous ultrasound-assisted ternary adsorption of dyes onto copper-doped zinc sulfide nanoparticles loaded on activated carbon: optimization by response surface methodology. Spectrochim ActaMol Biomol Spectrosc. 2015; 145: 203-212. doi: 10.1016/j.saa.2015.03.006

12. Azizi S, Ahmad MB, Namvar F, Mohamad R. Green biosynthesis and characterization of zinc oxide nanoparticles using brown marine macro alga Sargassummuticum aqueous extract. Mater Lett. 2014; 116: 275-277. doi: 10.1016/j.matlet.2013.11.038
13. Kalyani DC, Patil PS, Jadhav JP, Govindwar SP. Biodegradation of reactive textile dye Red BLI by an isolated bacterium Pseudomonas sp. SUK1. Biores Technol. 2008; 99(11): 4635-4641. doi: 10.1016/j.biortech.2007.06.058 